Nada M Al-Sayagh

BDS, MSc (Assist Proff)

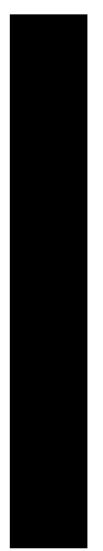

\section{Maxillary Arch Dimensional Changes in the Extraction and Non Extraction Orthodontic Treatment}

\author{
Dept of Pedod, orthod, and Prev Dentistry \\ College of Dentistry, University of Mosul
}

\begin{abstract}
Aims:To investigate whether extraction status and gender had any significant influence on the maxillary arch dimensions. Materials and Methods: A sample of 40 orthodontic patients (20 extraction and 20 non extraction) were included in this study. Males and females were evenly represented in both groups. All patients were treated with fixed edgewise appliances. Dental casts were taken before and after final orthodontic treatment, fifteen maxillary arch parameters were evaluated at pre - and post treatment stage and included; dental arch width at the canine, $1^{\text {st }}$ premolar, $2^{\text {nd }}$ premolar, $1^{\text {st }}$ molar (at mesiobuccal and distobuccal cusp tips), arch depth at canine and $1^{\text {st }}$ molar, arch lengths (incisal canine length, canine molar length and incisal molar length ), and arch perimeter. A paired sample t- test was used to evaluate the treatment changes in the extraction and non extraction groups and also to compare between males and females before treatment and after treatment. Results: Generally, in both genders, most pretreatment arch dimensions were not significantly different between extraction and non extraction groups, while after treatment the extraction treatment resulted in the reduction in the arch perimeters, arch depth, and arch length. Where as the non extraction group showed a significant increase in most maxillary arch dimensions. In addition both the extraction and non extraction treatment did not cause narrowing of the dental arch at the canine region. The direction of post treatment changes were similar in male and female subjects. However, the magnitude of the post treatment changes in some parameters differed significantly between females and males particularly in the non extraction group. Conclusions: the extraction and non extraction groups showed similar trend in some maxillary dimensions and different in other dimensions, thus it was concluded that the kind of treatment may affect the maxillary arch dimensions. In addition the non extraction group had a larger number of significant gender differences between females and males than the extraction group.
\end{abstract}

Key Words: Extraction, non extraction, maxillary arch dimensions.

Al-Sayagh NM. Maxillary Arch Dimensional Changes in the Extraction and Non Extraction Orthodontic Treatment. Al-Rafidain Dent J. 2008; 8(1): 26-37.

Received: 24/12/2006 Sent to Referees: 24/12/2006

Accepted for Publication: 23/4/2007

\section{INTRODUCTION}

Dental arch changes resulting from treatment are important to the orthodontist. An understanding of these changes is useful in treatment and retention planning by the clinician ${ }^{(1)}$.

Dental crowding and local irregularities are common causes of class I malocclusion. The two conventional orthodontic strategies used to resolve dental arch crowding are extraction and non - extraction ${ }^{(2)}$.

Many authors have compared samples of patient who have received extraction with those who have not ${ }^{(3-14)}$.

It has been suggested that the change in certain arch dimensions may be influenced by pre-treatment Angle classification and also extraction decision ${ }^{(5,8,15-19)}$. Studies vary in their description of exactly what these arch changes are. BeGole et $a l .{ }^{(16)}$ showed that there was a significant increase in the canine and premolar arch width during non - extraction treatment, but no such increase was seen in maxillary arches in cases where premolars were extracted. Other studies have shown a significant increase in intercanine and intermolar widths when treating with extrac- 
tion. Bishara et al., ${ }^{(18)}$ demonstrated a significantly greater increase in arch width at the anterior arch positions and at the premolars which were extracted during the treatment of class I and class II division 1 malocclusion than treatment without extraction. Kim and Gianelli ${ }^{(8)}$ also reported a significantly greater arch width increase in the extraction than in non extraction group. It has been reported, therefore that the intercanine distance can decrease, stay the same, or increase during extraction treatment; as compared to non - extraction treatment.

Cross et al., (19) found that the pretreatment arch dimensions were not significantly different among extraction and non - extraction groups, while after treatment the extraction cases showed a significant greater decrease in arch perimeter and arch length, But there were no significant different changes in arch width between extraction and non - extraction treatment.

At the start of treatment, the maxillary intercanine and intermolar widths of both extraction and non extraction groups didn't differ statistically. At the end of treatment the maxillary intercanine width of both groups increased significantly. The maxillary intermolar width increased significantly for the non - extraction group, the decreased in maxillary intermolar width for the extraction group was not significantly different ${ }^{(20)}$. Isik et al. ${ }^{(21)}$ also revealed that the distance between the upper canines was not affected by the treatment modality, upper premolar and molar arch widths increased more in the non extraction subjects when compared with those with extraction.

Although the literature has provided information regarding the effect of extraction and non - extraction therapy, the findings on the amount of dental arch changes of class I extraction and non - extraction therapy display variation. This may be attributed to the differing treatment modalities, malocclusion types, the degree of crowding, amount of overjet, presence of displaced canines and the variability in arch shape and sample sizes. Other influential factors that may modify treatment outcome are variations in the arch wire ${ }^{(20)}$.
Therefore, an attempt was made in this study to have a homogenous study group in terms of malocclusion type and treatment mechanics.

The purpose of this study was; (1) to determine the pre - and post - treatment maxillary arch dimensional changes in subjects treated with extraction and non extraction treatment, (2) to compare between extraction and non - extraction treatment concerning maxillary arch dimensions for males and females separately, and (3) to make a comparison of maxillary arch dimensions between male and female in extraction and non - extraction group at pretreatment and post treatment stage.

\section{MATERIALS AND METHODS}

A sample of 40 orthodontic patients (20 extraction and 20 non-extraction) was included in this study.

Males and females were evenly represented in both groups; the mean ages of the study groups at the beginning of orthodontic treatment was $14.5 \pm 2.7$ for the non - extraction group and $14.6 \pm 2.5$ for the extraction group. Mean treatment time was $21.5 \pm 7$ months for the non - extraction group and $27.8 \pm 8.2$ months for the extraction group.

The maxillary tooth size arch length discrepancies were $-3.9 \pm 1$. $\mathrm{mm}$ for the non- extraction group and $-6.9 \pm 2.0 \mathrm{~mm}$ for the extraction group.

All the patients who were treated by assistant professor Al-Sayagh at private clinic and all the patients who were treated by fixed edge wise technique 0.018" bracket slot were selected on the basis of the following criteria:

1. All patients had Angle class I malocclusion.

2. At the start of treatment, all patients were in the permanent dentition without any missing permanent teeth or congenitally absent teeth or significant facial asymmetries.

3. Non of the patients had any adjunctive appliance such as Quad Helix, a functional appliance or a rapid palatal expander used as part of their orthodontic treatment.

4. The patient whose treated involved ex- 
traction had undergone bilateral maxillary first premolar extraction as part of a comprehensive orthodontic treatment plan.

5. At the end of treatment, all cases achieved acceptable results.

6. Dental stone casts records were taken before and after final orthodontic treatment.

Dental vernier (Müncher model, Dentaurum 042 - 751, Germany) with fine tips measuring within $0.10 \mathrm{~mm}$ were used by one orthodontist to measure the following fifteen parameters including dental arch width, length, depth and perimeter on the maxillary pretreatment and post treatment dental cast:

1. Inter canine width (ICW): The distance between the cusp tips of the right and left canines ${ }^{(1,22,23)}$.

2. Inter first premolar width $\left(\mathrm{IP}_{1} \mathrm{~W}\right)$ : The distance between the buccal cusp tip of the right and left first premolars ${ }^{(24)}$.

3. Inter second premolar width $\left(\mathrm{IP}_{2} \mathrm{~W}\right)$ : The distance between the buccal cusp tip of the right and left second premolars ${ }^{(24)}$.

4. Inter molar width (IMW at MBCT):The distance between the mesiobuccal cusp tip of the right and left first permanent molars ${ }^{(24)}$.

5. Inter molar width (IMW at DBCT): The distance between the distobuccal cusp tip of the right and left first permanent molars ${ }^{(25)}$.

6. Canine vertical distance (C-VD): The vertical distance from the Inter incisal midline point to the inter canine distance at the cusp tips ${ }^{(26)}$.

7. Molar vertical distance (M-VD): The vertical distance from the Inter incisal midline point perpendicular to the inter molar distance at the mesiolingual cusp tips (MLCT) and at the distolingual cusp tips (DLCT) ${ }^{(26)}$.

8. Incisal - canine length (In-CL): The distance from the midpoint of the incisal edges of the central incirors to the canine cusp tip and for the right and left quadrants ${ }^{(26)}$.

9. Canine molar length (C-ML): The distance from canine cusp tip to the distobuccal cusp tip of the first permanent molar and for the right and left sides of the dental arch ${ }^{(25)}$.

10.Incisal molar length (In-ML):The linear distance from the Inter incisal midline point to the distobuccal cusp tip of the first molar and measured on the right and left sides of the dental arch (25)

11. Arch perimeters (A per): The sum of right and left In-CL and C-ML length.

All statistical analyses were performed using the SPSS soft ware package (SPSS for windows 98, version 10.0 SPSS Inc, Chicago).

For each variable, the arithmetic mean and standard deviation were calculated. A paired sample $\mathrm{t}$ - test was used to evaluate the treatment changes within the extraction and non extraction groups for both male and female subjects separately and also to compare males and females before and after treatment.

To test the reliability of the measurements, ten study dental casts were selected randomly and measured on a separate occasion by the same examiner. No statistically significant difference was found between the two measurements (paired t test, $p>0.05$ ).

\section{RESULTS}

Table (1) and (2) summarize the changes in the maxillary arch dimensions between pretreatment and post treatment in both extraction and non extraction groups respectively.

The extraction cases showed a significant decrease in the following parameters; IMW (MBCT), VMD, C-ML, In-ML and A per for female and male subjects. In addition, ICW was increased significantly in the female group.

The non extraction group showed a significant increased in $\mathrm{IP}_{2} \mathrm{~W}$, right In-CL and right In-ML in males and females, in addition the ICW, $\mathrm{IP}_{1} \mathrm{~W}$ and $\mathrm{A}$ per were significantly increased in the females group, while in the males there was a significant increase in the VMD and left InML. 
Table (1): Comparisons of maxillary arch dimensions between pretreatment and post treatment for females and males who were treated with extraction.

\begin{tabular}{|c|c|c|c|c|c|c|c|c|c|c|}
\hline \multirow[t]{2}{*}{ Gender } & \multicolumn{3}{|c|}{ Variables * } & \multicolumn{2}{|c|}{$\begin{array}{c}\text { Pretreatment } \\
\text { ( extraction cases) } \\
(\mathbf{n}=10)\end{array}$} & \multicolumn{2}{|c|}{$\begin{array}{l}\text { Post-treatment } \\
\text { (extraction cases) } \\
(\mathrm{n}=10)\end{array}$} & \multicolumn{2}{|c|}{ Difference } & \multirow[t]{2}{*}{$\mathbf{P}$ - value } \\
\hline & & & & Mean & SD & Mean & SD & Mean & SD & \\
\hline \multirow{14}{*}{ 苞 } & \multirow{4}{*}{ 尝 } & \multicolumn{2}{|c|}{ ICW } & 32.06 & 2.63 & 34.05 & 1.77 & -1.99 & 1.29 & $0.001^{*}$ \\
\hline & & \multicolumn{2}{|c|}{$\mathrm{IP}_{2} \mathrm{~W}$} & 42.72 & 2.17 & 42.10 & 1.28 & 0.62 & 1.87 & 0.321 \\
\hline & & \multicolumn{2}{|c|}{ IMW "MBCT" } & 48.99 & 2.16 & 46.73 & 1.69 & 2.26 & 2.59 & $0.022^{*}$ \\
\hline & & \multicolumn{2}{|c|}{ IMW "DBCT" } & 51.41 & 2.14 & 49.92 & 2.12 & 1.49 & 2.88 & 0.136 \\
\hline & \multirow{3}{*}{ 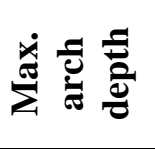 } & \multicolumn{2}{|c|}{ VCD } & 10.21 & 2.88 & 9.78 & 1.03 & .43 & 3.10 & 0.679 \\
\hline & & \multicolumn{2}{|c|}{ VMD "MLCT" } & 32.44 & 1.76 & 26.34 & 1.68 & 6.10 & 1.96 & $0.000^{*}$ \\
\hline & & \multicolumn{2}{|c|}{ VMD "DLCT" } & 36.35 & 1.57 & 30.65 & 1.71 & 5.70 & 2.11 & $0.000^{*}$ \\
\hline & \multirow{7}{*}{ 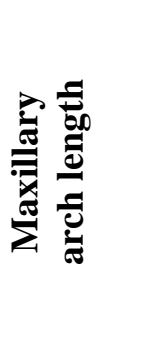 } & \multirow{2}{*}{ In-CL } & Right & 18.38 & 0.89 & 18.92 & 1.14 & -0.54 & 1.21 & 0.191 \\
\hline & & & Left & 18.61 & 1.97 & 19.49 & 1.16 & -.088 & 1.51 & 0.098 \\
\hline & & \multirow{2}{*}{ C-ML } & Right & 26.51 & 2.35 & 20.70 & 1.14 & 5.81 & 2.35 & $0.000 *$ \\
\hline & & & Left & 26.75 & 1.35 & 20.14 & 1.37 & 6.61 & 0.97 & $0.000^{*}$ \\
\hline & & \multirow{2}{*}{ In-ML } & Right & 42.22 & 0.86 & 36.74 & 1.61 & 5.48 & 1.77 & $0.000^{*}$ \\
\hline & & & Left & 41.82 & 2.14 & 37.46 & 3.00 & 4.36 & 2.23 & $0.000^{*}$ \\
\hline & & \multicolumn{2}{|c|}{ A per } & 89.55 & 3.53 & 79.27 & 4.23 & 10.28 & 3.10 & $0.000 *$ \\
\hline \multirow{14}{*}{$\frac{\stackrel{0}{\tilde{J}}}{\sum}$} & \multirow{4}{*}{ 苞 } & \multicolumn{2}{|c|}{ ICW } & 35.47 & 3.22 & 36.94 & 1.56 & -1.47 & 2.61 & 0.186 \\
\hline & & \multicolumn{2}{|c|}{$\mathrm{IP}_{2} \mathrm{~W}$} & 43.64 & 1.35 & 42.34 & 1.59 & 1.30 & 2.49 & 0.217 \\
\hline & & \multicolumn{2}{|c|}{ IMW "MBCT" } & 50.47 & 2.16 & 46.86 & 1.64 & 3.61 & 2.56 & $0.010^{*}$ \\
\hline & & \multicolumn{2}{|c|}{ IMW "DBCT" } & 52.76 & 2.18 & 50.39 & 2.03 & 2.37 & 2.78 & 0.064 \\
\hline & \multirow{3}{*}{ 离毞 } & \multicolumn{2}{|c|}{ VCD } & 10.96 & 0.72 & 9.99 & 0.80 & 0.97 & 0.45 & 0.127 \\
\hline & & VMD & $L C T^{\prime \prime}$ & 32.03 & 2.92 & 26.90 & 0.98 & 5.13 & 3.21 & $0.005^{*}$ \\
\hline & & VMD & $L C T^{\prime \prime}$ & 36.60 & 2.69 & 31.63 & 1.17 & 4.97 & 2.74 & $0.003^{*}$ \\
\hline & & In $C \mathrm{CI}$ & Right & 20.61 & 0.86 & 19.99 & 0.82 & 0.62 & 1.11 & $0.032 *$ \\
\hline & & & Left & 20.50 & 1.16 & 20.43 & 1.26 & 0.07 & 1.01 & 0.858 \\
\hline & ह0 & C MI & Right & 27.44 & 1.63 & 20.16 & 0.91 & 7.29 & 1.35 & $0.000 *$ \\
\hline & 灵 & $C-1 V I L$ & Left & 26.09 & 1.94 & 19.84 & 0.77 & 6.24 & 2.26 & $0.000^{*}$ \\
\hline & $\bar{\Sigma}$ & In & Right & 43.06 & 2.63 & 37.07 & 0.82 & 5.99 & 2.69 & $0.001 *$ \\
\hline & & $\mathrm{In}-\mathrm{M}$ & Left & 41.80 & 2.99 & 37.77 & 1.12 & 4.03 & 3.02 & $0.012 *$ \\
\hline & & & & 94.80 & 3.24 & 80.22 & 2.37 & 14.20 & 4.21 & $0.000^{*}$ \\
\hline
\end{tabular}

* All variables measured in millimeters.* Significant difference at $p<0.05$.

(ICW):Intercanine width; ( $\left.\mathrm{IP}_{2} \mathrm{~W}\right)$ : Inter second premolar width; (IMW at MBCT): Inter molar width at mesiobuccal cusp tip; (IMW at DBCT): Inter molar width at distobuccal cusp tip; (VCD): Vertical canine distance; (VMD) (MLCT): Vertical molar distance at the mesiolingual cusp tips; (VMD) (DLCT): Vertical molar distance at the distolingual cusp tips; (In-CL): Incisal-canine length; (C-ML): Canine molar length; (In-ML): Incisal - molar length; (A per): Arch perimeters. 
Table (2): Comparisons of maxillary arch dimensions between pretreatment and post treatment for females and males who were treated with non extraction.

\begin{tabular}{|c|c|c|c|c|c|c|c|c|c|c|}
\hline \multirow[t]{2}{*}{ Gender } & \multicolumn{3}{|c|}{ Variables $\star$} & \multicolumn{2}{|c|}{$\begin{array}{c}\text { Pretreatment } \\
\text { ( non extraction cases) } \\
(\mathbf{n}=10)\end{array}$} & \multicolumn{2}{|c|}{$\begin{array}{c}\text { Post-treatment } \\
\text { (non extraction cases) } \\
(\mathbf{n}=10)\end{array}$} & \multicolumn{2}{|c|}{ Difference } & \multirow[t]{2}{*}{ P - value } \\
\hline & & & & Mean & SD & Mean & SD & Mean & SD & \\
\hline \multirow{15}{*}{ 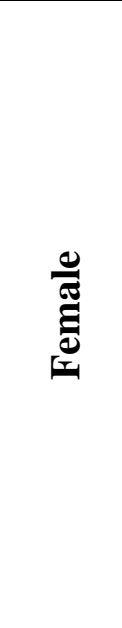 } & \multirow{5}{*}{ 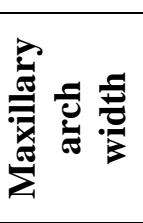 } & IC & & 34.43 & 1.30 & 36.73 & 0.59 & -2.30 & 0.85 & $0.001^{*}$ \\
\hline & & $\mathrm{IP}_{1}$ & & 39.83 & 0.89 & 42.63 & 0.58 & -2.80 & 1.48 & $0.006^{*}$ \\
\hline & & $\mathrm{IP}_{2}$ & & 43.27 & 2.46 & 46.33 & 0.49 & -3.07 & 2.89 & $0.048^{*}$ \\
\hline & & IMW "I & $B C T^{\prime \prime}$ & 48.30 & 3.16 & 50.03 & 1.74 & -1.73 & 3.64 & 0.296 \\
\hline & & IMW "I & $B C T^{\prime \prime}$ & 50.67 & 2.89 & 51.27 & 1.78 & -0.60 & 4.54 & 0.731 \\
\hline & \multirow{3}{*}{ 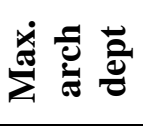 } & $\mathrm{VC}$ & & 9.60 & 1.02 & 9.73 & 0.60 & -1.33 & 0.49 & 0.537 \\
\hline & & VMD "I & $L C T^{\prime \prime}$ & 34.63 & 4.36 & 33.10 & 0.92 & 1.53 & 5.06 & 0.491 \\
\hline & & VMD " & $L C T^{\prime \prime}$ & 37.50 & 3.31 & 37.67 & 0.81 & -0.16 & 3.99 & 0.923 \\
\hline & \multirow{7}{*}{ 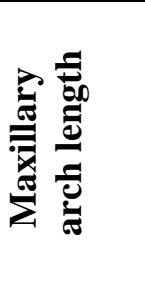 } & \multirow{2}{*}{ In-CL } & Right & 18.60 & 0.53 & 20.57 & 0.20 & -1.97 & 0.37 & $0.000 *$ \\
\hline & & & Left & 19.93 & 1.35 & 20.97 & 0.27 & -1.03 & 1.17 & 0.082 \\
\hline & & \multirow{2}{*}{ C-ML } & Right & 26.03 & 0.89 & 27.33 & 0.50 & -1.30 & 1.25 & $0.052^{*}$ \\
\hline & & & Left & 26.27 & 1.26 & 27.00 & 0.46 & -0.73 & 1.71 & 0.342 \\
\hline & & \multirow{3}{*}{\multicolumn{2}{|c|}{$\begin{array}{c}\text { In-ML } \begin{array}{r}\text { Right } \\
\text { Left }\end{array} \\
\text { A per }\end{array}$}} & 40.63 & 2.04 & 43.33 & 0.18 & -2.70 & 2.17 & $0.029 *$ \\
\hline & & & & 42.47 & 2.39 & 43.03 & 0.60 & -0.56 & 2.82 & 0.643 \\
\hline & & & & 90.83 & 3.81 & 95.77 & 0.52 & -4.93 & 4.93 & $0.035 *$ \\
\hline \multirow{15}{*}{$\frac{0}{\sum}$} & \multirow{5}{*}{ 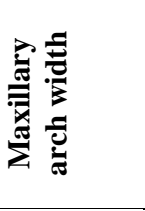 } & \multicolumn{2}{|c|}{ ICW } & 32.40 & 3.41 & 34.23 & 0.44 & -1.83 & 3.24 & 0.224 \\
\hline & & \multicolumn{2}{|c|}{$\mathrm{IP}_{1} \mathrm{~W}$} & 41.57 & 1.62 & 41.40 & 1.88 & 0.16 & 0.27 & 0.195 \\
\hline & & \multicolumn{2}{|c|}{$\mathrm{IP}_{2} \mathrm{~W}$} & 47.50 & 1.47 & 47.90 & 1.50 & -0.40 & 0.26 & $0.015^{*}$ \\
\hline & & \multirow{2}{*}{\multicolumn{2}{|c|}{$\begin{array}{l}\text { IMW "MBCT" } \\
\text { IMW "DBCT" }\end{array}$}} & 52.40 & 2.26 & 53.67 & 1.99 & -1.27 & 2.35 & 0.245 \\
\hline & & & & 55.70 & 2.10 & 55.50 & 1.59 & 0.20 & 0.79 & 0.565 \\
\hline & \multirow{3}{*}{ 这 휼 } & \multicolumn{2}{|c|}{ VCD } & 9.20 & 0.86 & 9.50 & 0.69 & -0.30 & 0.85 & 0.428 \\
\hline & & \multirow{2}{*}{\multicolumn{2}{|c|}{$\begin{array}{l}\text { VMD "MLCT" } \\
\text { VMD "DLCT" }\end{array}$}} & 31.33 & 1.37 & 32.83 & 1.14 & -1.50 & 0.55 & $0.001^{*}$ \\
\hline & & & & 36.37 & 1.61 & 37.57 & 1.13 & -1.20 & 0.70 & $0.009 *$ \\
\hline & \multirow{7}{*}{ 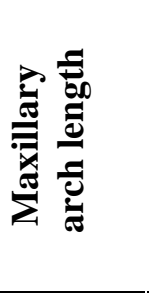 } & \multirow{2}{*}{ In-CL } & Right & 18.97 & 0.58 & 19.73 & 0.54 & -0.76 & 0.63 & $0.032 *$ \\
\hline & & & Left & 18.00 & 2.05 & 19.27 & 0.31 & -1.27 & 1.74 & 0.134 \\
\hline & & \multirow{2}{*}{ C-ML } & Right & 27.57 & 0.96 & 27.37 & 0.63 & 0.20 & 0.32 & 0.184 \\
\hline & & & Left & 27.83 & 0.31 & 27.57 & 0.10 & 0.26 & 0.33 & 0.112 \\
\hline & & \multirow{2}{*}{ In-ML } & Right & 43.50 & 0.26 & 44.20 & 0.62 & -0.70 & 0.17 & $0.000^{*}$ \\
\hline & & & Left & 42.83 & 0.64 & 44.10 & 0.55 & -1.27 & 0.76 & $0.010^{*}$ \\
\hline & & \multicolumn{2}{|c|}{ A per } & 92.70 & 2.11 & 93.93 & 1.16 & -1.23 & 3.53 & 0.398 \\
\hline
\end{tabular}

* All variables measured in millimeters.* Significant difference at $p<0.05$.

(ICW): Intercanine width; ( $\left.\mathrm{IP}_{1} \mathrm{~W}\right)$ : Inter first premolar width; $\left(\mathrm{IP}_{2} \mathrm{~W}\right)$ : Inter second premolar width; (IMW at MBCT): Inter molar width at mesiobuccal cusp tip; (IMW at DBCT): Inter molar width at destobuccal cusp tip; (VCD): Vertical canine distance; (VMD) (MLCT): Vertical molar distance at the mesiolingual cusp tips; (VMD) (DLCT): Vertical molar distance at the distolingual cusp tips; (In-CL): Incisal-canine length; (C-ML): Canine molar length; (In-ML): Incisal-molar length; (A per): Arch perimeters.

The comparisons between the extraction and non extraction group for both females and males at the pretreatment and post treatment stages are demonstrated in Tables (3) and (4) respectively. At the pretreatment stage, there were no significant difference between extraction and non extraction group in all measurements except the IMW (DBCT),VCD and right In-CL in males group, while after treatment, the non extraction group revealed a higher mean value for all measurements except the ICW, VCD in females and males groups and also $\mathrm{IP}_{2} \mathrm{~W}$, IMW (MBCT), InCL and VMD (MLCT) in males, and IMW (DBCT) in females which were not significantly different. 
Table (3): Comparisons of maxillary arch dimensions between extraction and non extraction groups for females and males at pretreatment stage.

\begin{tabular}{|c|c|c|c|c|c|c|c|c|c|c|}
\hline \multirow[t]{2}{*}{ Gender } & \multicolumn{3}{|c|}{ Variables } & \multicolumn{2}{|c|}{$\begin{array}{c}\text { Pretreatment } \\
\text { (extraction cases) } \\
(\mathbf{n}=10)\end{array}$} & \multicolumn{2}{|c|}{$\begin{array}{c}\text { Pretreatment } \\
\text { (non extraction cases) } \\
(\mathbf{n}=10)\end{array}$} & \multicolumn{2}{|c|}{ Difference } & \multirow[t]{2}{*}{$P$ - value } \\
\hline & & & & Mean & SD & Mean & SD & Mean & SD & \\
\hline \multirow{15}{*}{ 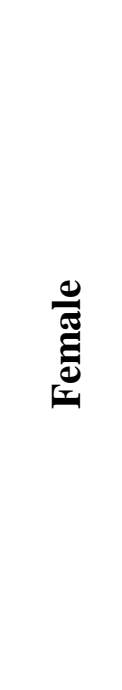 } & \multirow{5}{*}{ 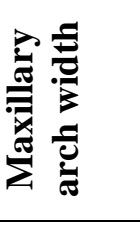 } & \multicolumn{2}{|c|}{ ICW } & 32.06 & 2.63 & 34.43 & 1.30 & -2.37 & 3.37 & 0.263 \\
\hline & & \multicolumn{2}{|c|}{$\mathrm{IP}_{1} \mathrm{~W}$} & 37.20 & 2.01 & 39.83 & 0.89 & -2.63 & 2.77 & 0.122 \\
\hline & & \multicolumn{2}{|c|}{$\mathrm{IP}_{2} \mathrm{~W}$} & 42.72 & 2.17 & 43.27 & 2.46 & -0.55 & 4.14 & 0.586 \\
\hline & & \multirow{2}{*}{\multicolumn{2}{|c|}{$\begin{array}{l}\text { IMW "MBCT" } \\
\text { IMW "DBCT" }\end{array}$}} & 48.99 & 2.16 & 48.30 & 3.16 & 0.69 & 3.89 & 0.958 \\
\hline & & & & 51.41 & 2.14 & 50.67 & 2.89 & 0.74 & 3.89 & 0.819 \\
\hline & \multirow{3}{*}{ 离 듈 흘 } & \multicolumn{2}{|c|}{ VCD } & 10.21 & 2.88 & 9.60 & 1.02 & 0.61 & 3.89 & 0.551 \\
\hline & & \multicolumn{2}{|c|}{ VMD "MLCT" } & 32.44 & 1.76 & 34.63 & 4.36 & -2.19 & 3.71 & 0.317 \\
\hline & & \multicolumn{2}{|c|}{ VMD "DLCT" } & 36.35 & 1.57 & 37.50 & 3.31 & -1.15 & 3.45 & 0.458 \\
\hline & \multirow{7}{*}{ 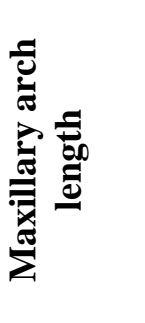 } & \multirow{2}{*}{ In-CL } & Right & 18.38 & 0.89 & 18.60 & 0.53 & -0.22 & 0.93 & 0.383 \\
\hline & & & Left & 18.61 & 1.97 & 19.93 & 1.35 & -1.32 & 2.42 & 0.082 \\
\hline & & \multirow{2}{*}{ C-ML } & Right & 26.51 & 2.35 & 26.03 & 0.89 & 0.48 & 1.77 & 0.238 \\
\hline & & & Left & 26.75 & 1.35 & 26.27 & 1.26 & 0.48 & 1.13 & 0.733 \\
\hline & & \multirow{2}{*}{ In-ML } & Right & 42.22 & 0.86 & 40.63 & 2.04 & 1.59 & 2.83 & 0.275 \\
\hline & & & Left & 41.82 & 2.14 & 42.47 & 2.39 & -1.25 & 2.97 & 0.349 \\
\hline & & \multicolumn{2}{|c|}{ A per } & 89.55 & 3.53 & 90.83 & 3.81 & -1.28 & 5.25 & 0.547 \\
\hline \multirow{15}{*}{$\frac{0}{\sum}$} & \multirow{5}{*}{ 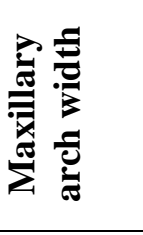 } & \multicolumn{2}{|c|}{ ICW } & 35.47 & 3.22 & 32.40 & 3.41 & 3.07 & 2.90 & 0.067 \\
\hline & & \multicolumn{2}{|c|}{$\mathrm{IP}_{1} \mathrm{~W}$} & 39.37 & 2.89 & 41.57 & 1.62 & -2.2 & 3.27 & 0.364 \\
\hline & & \multicolumn{2}{|c|}{$\mathrm{IP}_{2} \mathrm{~W}$} & 43.64 & 1.35 & 47.50 & 1.47 & -3.86 & 1.46 & 0.050 \\
\hline & & \multicolumn{2}{|c|}{ IMW "MBCT" } & 50.47 & 2.16 & 52.40 & 2.26 & -1.93 & 1.81 & 0.175 \\
\hline & & \multicolumn{2}{|c|}{ IMW "DBCT" } & 52.76 & 2.18 & 55.70 & 2.10 & -2.94 & 0.40 & $0.005 *$ \\
\hline & & & & 10.96 & 0.72 & 9.20 & 0.86 & 1.76 & 0.20 & $0.008^{*}$ \\
\hline & 를 들 & VMD ' & $L C T^{\prime \prime}$ & 32.03 & 2.92 & 31.33 & 1.37 & -0.7 & 5.69 & 0.796 \\
\hline & < & VMD & $L C T^{\prime \prime}$ & 36.60 & 2.69 & 36.37 & 1.61 & 0.23 & 5.61 & 0.842 \\
\hline & & In-CI & Right & 20.61 & 0.86 & 18.97 & 0.58 & 1.64 & 0.99 & $0.008 *$ \\
\hline & & 111-CL & Left & 20.50 & 1.16 & 18.00 & 2.05 & 2.5 & 2.75 & 0.071 \\
\hline & $\pi=$ & C-MI & Right & 27.44 & 1.63 & 27.57 & 0.96 & -0.13 & 2.53 & 0.976 \\
\hline & 트 & $c-1 V I L$ & Left & 26.09 & 1.94 & 27.83 & 0.31 & -1.74 & 2.09 & 0.087 \\
\hline & $\bar{x}$ & In-M & Right & 43.06 & 2.63 & 43.50 & 0.26 & -0.44 & 3.74 & 0.680 \\
\hline & $\sum^{\pi}$ & & Left & 41.80 & 2.99 & 42.83 & 0.64 & -1.03 & 4.91 & 0.804 \\
\hline & & & & 94.80 & 3.24 & 92.70 & 2.11 & 2.1 & 4.87 & 0.339 \\
\hline
\end{tabular}

*All variables measured in millimeters. *Significant difference at $\mathrm{p}<0.05$.

(ICW): Intercanine width; ( $\left.\mathrm{IP}_{1} \mathrm{~W}\right)$ : Inter first premolar width; $\left(\mathrm{IP}_{2} \mathrm{~W}\right)$ : Inter second premolar width; (IMW at MBCT): Inter molar width at mesiobuccal cusp tip; (IMW at DBCT): Inter molar width at destobuccal cusp tip; (VCD): Vertical canine distance; (VMD) (MLCT): Vertical molar distance at the mesiolingual cusp tips; (VMD) (DLCT): Vertical molar distance at the distolingual cusp tips; (In-CL): Incisal-canine length; (C-ML): Canine molar length; (In-ML): Incisal-molar length; (A per): Arch perimeters. 
Table (4): Comparisons of maxillary arch dimensions between extraction and non extraction groups for females and males at post treatment stage.

\begin{tabular}{|c|c|c|c|c|c|c|c|c|c|c|}
\hline \multirow[t]{2}{*}{ Gender } & \multirow{2}{*}{\multicolumn{3}{|c|}{ Variables * }} & \multicolumn{2}{|c|}{$\begin{array}{c}\text { Post-treatment } \\
\text { ( extraction cases) } \\
(\mathbf{n}=10)\end{array}$} & \multicolumn{2}{|c|}{$\begin{array}{c}\text { Post-treatment } \\
\text { (non extraction cases) } \\
(\mathbf{n}=10)\end{array}$} & \multicolumn{2}{|c|}{ Difference } & \multirow[t]{2}{*}{ P - value } \\
\hline & & & & Mean & SD & Mean & SD & Mean & SD & \\
\hline \multirow{14}{*}{ 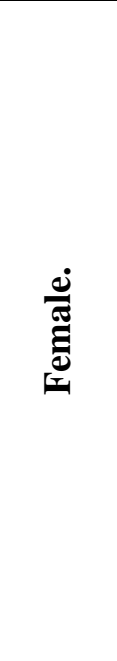 } & \multirow{4}{*}{ 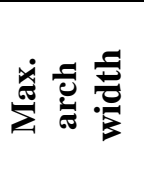 } & \multicolumn{2}{|c|}{ ICW } & 34.05 & 1.77 & 36.73 & 0.59 & -2.68 & 3.27 & 0.723 \\
\hline & & \multicolumn{2}{|c|}{$\mathrm{IP}_{2} \mathrm{~W}$} & 42.10 & 1.28 & 46.33 & 0.49 & -4.23 & 2.09 & $0.011^{*}$ \\
\hline & & \multicolumn{2}{|c|}{ IMW "MBCT" } & 46.73 & 1.69 & 50.03 & 1.74 & -3.3 & 1.70 & $0.037 *$ \\
\hline & & \multicolumn{2}{|c|}{ IMW "DBCT" } & 49.92 & 2.12 & 51.27 & 1.78 & -1.35 & 1.54 & 0.110 \\
\hline & \multirow{3}{*}{ 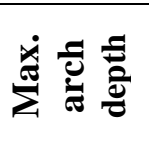 } & \multirow{3}{*}{\multicolumn{2}{|c|}{$\begin{array}{c}\text { VCD } \\
\text { VMD "MLCT" } \\
\text { VMD "DLCT" }\end{array}$}} & 9.78 & 1.03 & 9.73 & 0.60 & 0.05 & 1.36 & 0.108 \\
\hline & & & & 26.34 & 1.68 & 33.10 & 0.92 & -6.76 & 2.68 & $0.005 *$ \\
\hline & & & & 30.65 & 1.71 & 37.67 & 0.81 & -7.02 & 2.83 & $0.004^{*}$ \\
\hline & \multirow{7}{*}{ 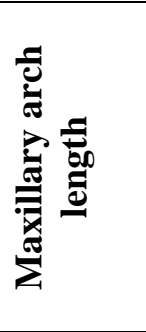 } & \multirow{2}{*}{ In-CL } & Right & 18.92 & 1.05 & 20.57 & 0.20 & -1.65 & 1.17 & $0.010^{*}$ \\
\hline & & & Left & 19.49 & 1.08 & 20.97 & 0.27 & -1.48 & 1.04 & $0.008 *$ \\
\hline & & \multirow{2}{*}{ C-ML } & Right & 20.70 & 1.16 & 27.33 & 0.50 & -6.63 & 1.09 & $0.000 *$ \\
\hline & & & Left & 20.14 & 1.10 & 27.00 & 0.46 & -6.86 & 1.25 & $0.000 *$ \\
\hline & & \multirow{2}{*}{ In-ML } & Right & 36.74 & 1.81 & 43.33 & 0.18 & -6.59 & 2.48 & $0.010^{*}$ \\
\hline & & & Left & 37.46 & 1.75 & 43.03 & 0.60 & -5.57 & 2.64 & $0.005^{*}$ \\
\hline & & \multicolumn{2}{|c|}{ A per } & 79.27 & 4.23 & 95.77 & 0.52 & -16.5 & 3.97 & $0.000 *$ \\
\hline \multirow{14}{*}{$\frac{0}{\sum}$} & & \multicolumn{2}{|c|}{ ICW } & 36.94 & 1.56 & 34.23 & 0.44 & 2.71 & 0.61 & 0.053 \\
\hline & 흘 돌 올 & \multicolumn{2}{|c|}{$\mathrm{IP}_{2} \mathrm{~W}$} & 42.34 & 1.59 & 47.90 & 1.47 & -5.56 & 2.35 & 0.068 \\
\hline & $\stackrel{x}{z}$ & \multicolumn{2}{|c|}{ IMW "MBCT" } & 46.86 & 1.64 & 53.67 & 2.26 & -6.81 & 2.56 & 0.050 \\
\hline & & \multicolumn{2}{|c|}{ IMW "DBCT" } & 50.39 & 2.03 & 55.50 & 2.10 & -5.11 & 1.55 & $0.047^{*}$ \\
\hline & \multirow{3}{*}{ 츌 흘 을 } & \multicolumn{2}{|c|}{ VCD } & 9.99 & 0.80 & 9.50 & 0.86 & 0.49 & 1.61 & 0.394 \\
\hline & & \multicolumn{2}{|c|}{ VMD "MLCT" } & 26.90 & 0.98 & 32.83 & 1.37 & -5.93 & 2.65 & 0.059 \\
\hline & & \multicolumn{2}{|c|}{ VMD "DLCT" } & 31.63 & 1.17 & 37.57 & 1.61 & -5.94 & 2.48 & $0.048 *$ \\
\hline & & & Right & 19.99 & 0.82 & 19.73 & 0.54 & 0.26 & 1.12 & 0.809 \\
\hline & 른 & & Left & 20.43 & 1.26 & 19.27 & 0.31 & 1.16 & 1.41 & 0.107 \\
\hline & $\vec{\nabla}=$ & & Right & 20.16 & 0.91 & 27.37 & 0.63 & -7.21 & 1.56 & $0.000 *$ \\
\hline & 可 & $\mathrm{C}-\mathrm{MIL}$ & Left & 19.84 & 0.77 & 27.57 & 0.10 & -7.73 & 0.90 & $0.000 *$ \\
\hline & 름 & & Right & 37.07 & 0.82 & 44.20 & 0.62 & -7.13 & 1.31 & $0.011^{*}$ \\
\hline & $\sum^{\pi}$ & III-IVIL & Left & 37.77 & 1.12 & 44.10 & 0.55 & -6.33 & 0.66 & $0.004^{*}$ \\
\hline & & & & 80.22 & 2.37 & 93.93 & 1.16 & -13.71 & 3.53 & $0.000^{*}$ \\
\hline
\end{tabular}

* All variables measured in millimeters.* Significant difference at $p<0.05$.

(ICW): Intercanine width; $\left(\mathrm{IP}_{2} \mathrm{~W}\right)$ : Inter second premolar width; (IMW at MBCT): Inter molar width at mesiobuccal cusp tip; (IMW at DBCT): Inter molar width at destobuccal cusp tip; (VCD): Vertical canine distance; (VMD) (MLCT): Vertical molar distance at the mesiolingual cusp tips; (VMD)

(DLCT): Vertical molar distance at the distolingual cusp tips; (In-CL): Incisal-canine length; (C-ML): Canine molar length; (In-ML): Incisal-molar length; (A per): Arch perimeters.

Comparison between females and males in the extraction group Table (5) and non extraction group Table (6) indicated that in the extraction group there were no significant differences in all maxillary arch dimensions except in $\mathrm{IP}_{1} \mathrm{~W}$, left $\mathrm{C}-\mathrm{ML}$ and A per which were significantly higher in males than females at the pretreatment stage. While in the non extrac- tion group, males had a higher mean values than females in the following measurements; $\mathrm{IP}_{1} \mathrm{~W}, \mathrm{IP}_{2} \mathrm{~W}$, IMW (DBCT), left C-ML and In-ML at the pre treatment stage, but after treatment, the females had a higher mean value than males in the $\mathrm{IP}_{1} \mathrm{~W}, \mathrm{VMD}$, In-CL where as the males had a higher mean value of right $\mathrm{C}-\mathrm{ML}$ and left In-ML than females. 
Table (5): Comparisons of maxillary arch dimensions between females and males in extraction group at pretreatment and post treatment stage.

\begin{tabular}{|c|c|c|c|c|c|c|c|c|c|c|}
\hline \multirow[t]{2}{*}{ Stage } & \multirow{2}{*}{\multicolumn{3}{|c|}{ Variables }} & \multicolumn{2}{|c|}{$\begin{array}{c}\text { Female } \\
(n=10)\end{array}$} & \multicolumn{2}{|c|}{$\begin{array}{c}\text { Male } \\
(\mathbf{n}=10)\end{array}$} & \multicolumn{2}{|c|}{ Difference } & \multirow{2}{*}{$\begin{array}{c}\mathbf{P}- \\
\text { value }\end{array}$} \\
\hline & & & & Mean & SD & Mean & SD & Mean & SD & \\
\hline \multirow{15}{*}{ 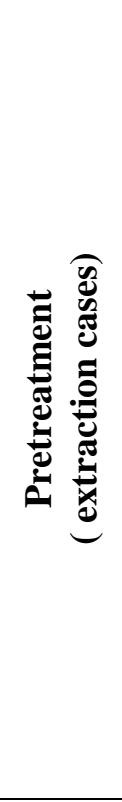 } & \multirow{5}{*}{ 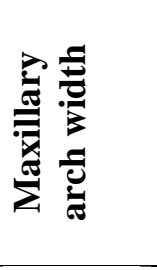 } & \multicolumn{2}{|c|}{ ICW } & 32.06 & 2.63 & 35.47 & 3.22 & -3.41 & 3.25 & 0.460 \\
\hline & & \multicolumn{2}{|c|}{$\mathrm{IP}_{1} \mathrm{~W}$} & 37.20 & 2.01 & 39.37 & 2.89 & -2.17 & 4.43 & $0.011^{*}$ \\
\hline & & \multicolumn{2}{|c|}{$\mathrm{IP}_{2} \mathrm{~W}$} & 42.72 & 2.17 & 43.64 & 1.35 & -0.92 & 3.56 & 0.117 \\
\hline & & \multicolumn{2}{|c|}{ IMW "MBCT" } & 48.99 & 2.16 & 50.47 & 2.16 & -1.48 & 3.96 & 0.411 \\
\hline & & \multicolumn{2}{|c|}{ IMW "DBCT" } & 51.41 & 2.14 & 52.76 & 2.18 & -1.35 & 2.71 & 0.584 \\
\hline & & \multicolumn{2}{|c|}{ VCD } & 10.21 & 2.88 & 10.96 & 0.72 & 0.75 & 2.96 & 0.545 \\
\hline & 突 & \multicolumn{2}{|c|}{ VMD "MLCT" } & 32.44 & 1.76 & 32.03 & 2.92 & 0.41 & 3.69 & 0.510 \\
\hline & & \multicolumn{2}{|c|}{ VMD "DLCT" } & 36.35 & 1.57 & 36.60 & 2.69 & -0.25 & 3.47 & 0.310 \\
\hline & \multirow{7}{*}{ 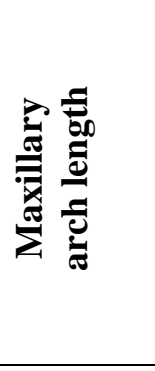 } & \multirow{2}{*}{ In-CL } & Right & 18.38 & 0.89 & 20.61 & 0.86 & -2.23 & 1.14 & 0.694 \\
\hline & & & Left & 18.61 & 1.97 & 20.50 & 1.16 & -1.89 & 1.62 & 0.559 \\
\hline & & \multirow{2}{*}{ C-ML } & Right & 26.51 & 2.35 & 27.44 & 1.63 & -0.93 & 3.07 & 0.997 \\
\hline & & & Left & 26.75 & 1.35 & 26.09 & 1.94 & 0.66 & 3.07 & $0.020^{*}$ \\
\hline & & \multirow{2}{*}{ In-MD } & Right & 42.22 & 0.86 & 43.06 & 2.63 & -0.84 & 2.76 & 0.904 \\
\hline & & & Left & 41.82 & 2.14 & 41.80 & 2.99 & -0.02 & 3.15 & 0.949 \\
\hline & & \multicolumn{2}{|c|}{ A per } & 89.55 & 3.53 & 94.80 & 3.24 & -5.25 & 5.40 & $0.032^{*}$ \\
\hline \multirow{14}{*}{ 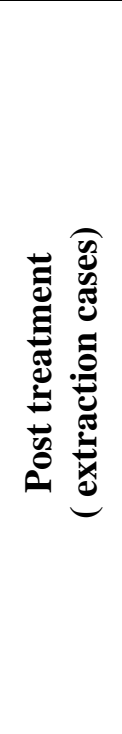 } & \multirow{4}{*}{ 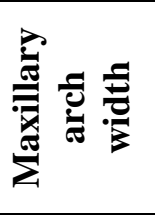 } & \multicolumn{2}{|c|}{ ICW } & 34.05 & 1.77 & 36.94 & 1.56 & -2.89 & 2.03 & 0.578 \\
\hline & & \multicolumn{2}{|c|}{$\mathrm{IP}_{2} \mathrm{~W}$} & 42.10 & 1.28 & 42.34 & 1.59 & -0.24 & 1.98 & 0.902 \\
\hline & & \multicolumn{2}{|c|}{ IMW "MBCT" } & 46.73 & 1.69 & 46.86 & 1.64 & -0.13 & 2.95 & 0.222 \\
\hline & & \multicolumn{2}{|c|}{ IMW "DBCT" } & 49.92 & 2.12 & 50.39 & 2.03 & -0.47 & 3.55 & 0.301 \\
\hline & & \multicolumn{2}{|c|}{ VCD } & 9.78 & 1.03 & 9.99 & 0.80 & -0.21 & 1.41 & 0.666 \\
\hline & 気 & VMD & $L C T^{\prime \prime}$ & 26.34 & 1.68 & 26.90 & 0.98 & -0.56 & 1.83 & 0.841 \\
\hline & & VMD & $L C T^{\prime \prime}$ & 30.65 & 1.71 & 31.63 & 1.17 & -0.98 & 2.12 & 0.854 \\
\hline & & & Right & 18.92 & 1.05 & 19.99 & 0.82 & -1.07 & 1.45 & 0.534 \\
\hline & & III-CL & Left & 19.49 & 1.08 & 20.43 & 1.26 & -0.94 & 1.39 & 0.586 \\
\hline & 氞 & C-MI & Right & 20.70 & 1.16 & 20.16 & 0.91 & -0.54 & 1.58 & 0.531 \\
\hline & 可 & $C-I V I L$ & Left & 20.14 & 1.10 & 19.84 & 0.77 & 0.3 & 1.02 & 0.409 \\
\hline & $\sum^{\pi}$ & & Right & 36.74 & 1.81 & 37.07 & 0.82 & -0.33 & 2.22 & 0.198 \\
\hline & & & Left & 37.46 & 1.75 & 37.77 & 1.12 & -0.31 & 2.05 & 0.810 \\
\hline & & & & 79.27 & 4.23 & 80.22 & 2.37 & -0.95 & 3.97 & .0127 \\
\hline
\end{tabular}

* All variables measured in millimeters. * Significant difference at $p<0.05$.

(ICW): Intercanine width; $\left(\mathrm{IP}_{1} \mathrm{~W}\right)$ : Inter first premolar width; $\left(\mathrm{IP}_{2} \mathrm{~W}\right)$ : Inter second premolar width; (IMW at MBCT): Inter molar width at mesiobuccal cusp tip; (IMW at DBCT): Inter molar width at destobuccal cusp tip; (VCD): Vertical canine distance; (VMD) (MLCT): Vertical molar distance at the mesiolingual cusp tips; (VMD) (DLCT): Vertical molar distance at the distolingual cusp tips; (In-CL): Incisal-canine length; (C-ML): Canine molar length; (In-ML): Incisal-molar length; (A per): Arch perimeters. 
Table (6): Comparisons of maxillary arch dimensions between females and males in non extraction group at pretreatment and post treatment stage.

\begin{tabular}{|c|c|c|c|c|c|c|c|c|c|c|}
\hline \multirow[t]{2}{*}{ Stage } & \multirow{2}{*}{\multicolumn{3}{|c|}{ Variables }} & \multicolumn{2}{|c|}{$\begin{array}{l}\text { Female } \\
(n=10)\end{array}$} & \multicolumn{2}{|c|}{$\begin{array}{c}\text { Male } \\
(n=10)\end{array}$} & \multicolumn{2}{|c|}{ Difference } & \multirow{2}{*}{$\begin{array}{c}\mathbf{P}- \\
\text { value }\end{array}$} \\
\hline & & & & Mean & SD & Mean & SD & Mean & SD & \\
\hline \multirow{15}{*}{ 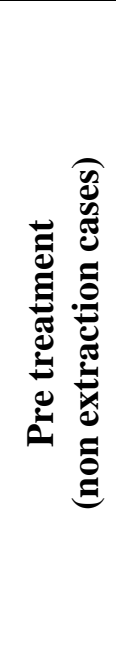 } & \multirow{5}{*}{ 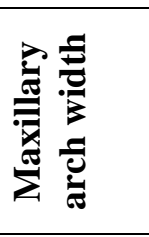 } & \multicolumn{2}{|c|}{ ICW } & 34.43 & 1.30 & 32.40 & 3.41 & 2.03 & 4.38 & 0.158 \\
\hline & & \multicolumn{2}{|c|}{$\mathrm{IP}_{1} \mathrm{~W}$} & 39.83 & 0.89 & 41.57 & 1.62 & -1.73 & 1.04 & $0.052 *$ \\
\hline & & \multicolumn{2}{|c|}{$\mathrm{IP}_{2} \mathrm{~W}$} & 43.27 & 2.46 & 47.50 & 1.47 & -4.23 & 0.98 & $0.000 *$ \\
\hline & & \multicolumn{2}{|c|}{ IMW "MBCT" } & 48.30 & 3.16 & 52.40 & 2.26 & -4.10 & 3.79 & 0.928 \\
\hline & & \multicolumn{2}{|c|}{ IMW "DBCT" } & 50.67 & 2.89 & 55.70 & 2.10 & -5.03 & 0.81 & $0.000^{*}$ \\
\hline & \multirow{3}{*}{ 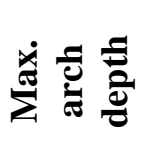 } & \multicolumn{2}{|c|}{ VCD } & 9.60 & 1.02 & 9.20 & 0.86 & 0.40 & 0.92 & 0.290 \\
\hline & & \multicolumn{2}{|c|}{ VMD "MLCT" } & 34.63 & 4.36 & 31.33 & 1.37 & 3.30 & 5.10 & 0.402 \\
\hline & & \multicolumn{2}{|c|}{ VMD "DLCT" } & 37.50 & 3.31 & 36.37 & 1.61 & 1.13 & 4.68 & 0.660 \\
\hline & \multirow{7}{*}{ 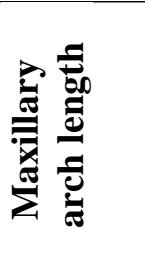 } & \multirow{2}{*}{\multicolumn{2}{|c|}{$\begin{array}{lc}\text { In-CL } & \text { Right } \\
\text { Left }\end{array}$}} & 18.60 & 0.53 & 18.97 & 0.58 & -0.36 & 0.58 & 0.357 \\
\hline & & & & 19.93 & 1.35 & 18.00 & 2.05 & 1.93 & 3.15 & 0.113 \\
\hline & & \multirow{2}{*}{ C-ML } & Right & 26.03 & 0.89 & 27.57 & 0.96 & -1.53 & 1.66 & 0.198 \\
\hline & & & Left & 26.27 & 1.26 & 27.83 & 0.31 & -1.57 & 1.57 & $0.001 *$ \\
\hline & & \multirow{2}{*}{\multicolumn{2}{|c|}{$\begin{array}{cc}\text { In-ML } & \text { Right } \\
\text { Left }\end{array}$}} & 40.63 & 2.04 & 43.50 & 0.62 & -2.87 & 2.66 & $0.000 *$ \\
\hline & & & & 42.47 & 2.39 & 42.83. & 0.64 & -0.36 & 3.01 & $0.005^{*}$ \\
\hline & & \multicolumn{2}{|c|}{ A per } & 90.83 & 3.81 & 92.70 & 2.11 & -1.87 & 5.72 & 0.460 \\
\hline \multirow{15}{*}{ 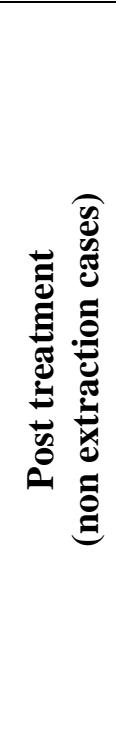 } & \multirow{5}{*}{ 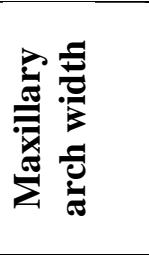 } & \multicolumn{2}{|c|}{ ICW } & 36.73 & 0.59 & 34.23 & 0.44 & 2.50 & 0.35 & 0.059 \\
\hline & & \multicolumn{2}{|c|}{$\mathrm{IP}_{1} \mathrm{~W}$} & 42.63 & 0.58 & 41.40 & 1.87 & 1.23 & 2.38 & $0.037^{*}$ \\
\hline & & \multicolumn{2}{|c|}{$\mathrm{IP}_{2} \mathrm{~W}$} & 46.33 & 0.49 & 47.90 & 1.50 & -1.57 & 1.91 & 0.069 \\
\hline & & \multicolumn{2}{|c|}{ IMW "MBCT" } & 50.03 & 1.74 & 53.67 & 0.98 & -3.63 & 3.09 & 0.478 \\
\hline & & IMW " & $B C T^{\prime \prime}$ & 51.27 & 1.77 & 55.50 & 0.59 & -4.23 & 3.18 & 0.065 \\
\hline & & $\mathrm{VC}$ & & 9.73 & 0.60 & 9.50 & 0.69 & 0.23 & 1.02 & 0.654 \\
\hline & 気 & VMD " & {$\left[L C T^{\prime \prime}\right.$} & 33.10 & 0.92 & 32.83 & 1.14 & 0.26 & 0.22 & $0.000 *$ \\
\hline & & VMD ' & $L C T^{\prime \prime}$ & 37.67 & 0.81 & 37.57 & 1.13 & 0.01 & 0.47 & $0.006^{*}$ \\
\hline & & & Right & 20.57 & 0.20 & 19.73 & 0.54 & 0.83 & 0.74 & $0.004 *$ \\
\hline & & & Left & 20.97 & 0.27 & 19.27 & 0.31 & 1.70 & 0.58 & $0.000 *$ \\
\hline & 츠 & & Right & 27.33 & 0.50 & 27.37 & 0.63 & -0.03 & 0.13 & $0.000^{*}$ \\
\hline & $\bar{\nabla}$ & $\mathrm{C}-\mathrm{ML}$ & Left & 27.00 & 0.46 & 27.57 & 0.10 & -0.57 & 0.42 & 0.313 \\
\hline & $\sum^{\pi}$ & & Right & 43.30 & 0.18 & 44.20 & 0.62 & -0.86 & 0.49 & 0.062 \\
\hline & & In-ML & Left & 43.03 & 0.60 & 44.10 & 0.55 & -1.07 & 1.12 & $0.039 *$ \\
\hline & & A & & 95.77 & 0.52 & 93.93 & 1.16 & 1.83 & 0.81 & 0.058 \\
\hline
\end{tabular}

* All variables measured in millimeters. * Significant difference at $p<0.05$.

(ICW): Intercanine width; $\left(\mathrm{IP}_{1} \mathrm{~W}\right)$ : Inter first premolar width; $\left(\mathrm{IP}_{2} \mathrm{~W}\right)$ : Inter second premolar width; (IMW at MBCT): Inter molar width at mesiobuccal cusp tip; (IMW at DBCT): Inter molar width at destobuccal cusp tip; (VCD): Vertical canine distance; (VMD) (MLCT): Vertical molar distance at the mesiolingual cusp tips; (VMD) (DLCT): Vertical molar distance at the distolingual cusp tips; (In-CL): Incisal-canine length; (C-ML): Canine molar length; (In-ML): Incisal-molar length; (A per): Arch perimeters.

\section{DISCUSSION}

Some researches have documented that arch dimensional changes occur both with the orthodontic treatment after the extraction of teeth and with the non extraction therapy ${ }^{(22,23)}$.

In this study, generally when comparing the end and start points of treatment with extraction Table (1), all measure- ments demonstrated the reduction except the ICW which significantly increased in female subjects. It has been suggested that in extraction cases, the canines could be moved to the buccal, if they were moved distally into the extraction site, thereby occupying a wider part of the arch ${ }^{(27)}$. Other studies also supported these findings demonstrating increases in the maxillary 
canine width ${ }^{(12,20,28)}$.

The increase in the maxillary inter canine and inter premolar and width for the non extraction patients as demonstrated in Table (2) can be explained by minimal expansion with the arch wires.

The increased maxillary inter molar width in the non extraction group was not significant but in the extraction group, the IMW (MBCT) was significantly decreased. The inter canine and inter molar width findings are similar to the findings of other studies ${ }^{(19,22,31)}$. Another important consideration in arch widths is the tooth size arch length discrepancy ${ }^{(9,22,29)}$. In the study of Aksu and Kocadereli ${ }^{(20)}$, there was more crowding in the extraction group than in non extraction group and they found that after extraction treatment the posterior teeth moved mesially into narrower parts of the arch, indicating that anchorage requirement were kept moderate. In the non extraction group, because of less tooth size arch length discrepancy, the crowding might be treated mostly by the movement of the anterior teeth. The result of this study supported these findings but this disagreement with other studies $(6,7,17)$ who found that tooth size arch length discrepancy not to have any effect on dental arch width changes.

Extraction cases showed a significant greater decrease in arch depth and arch length particularly posterior arch length (C-ML) and arch perimeter, these findings in accordance with other studies ${ }^{(19,29)}$.

Generally, most pretreatment arch dimensions were not significantly different between extraction and non extraction group Table (3), while after treatment, the non extraction group had a higher mean values in most measurements Table (4), this indicated that the kind of treatment may affect on the maxillary arch dimensions.

Comparisons between male and female subjects Table (5) indicated that although the males in the extraction group had a higher mean value than females in various maxillary arch dimensions, but these were not significant except in $\mathrm{IP}_{1} \mathrm{~W}$, left C-ML and A per which were significantly larger in males at the pretreatment stage, while the non extraction group Table (6) had a larger number of significant difference between females and males than the extraction group, this may attributed to the malocclusion which was sever in the extraction group and these findings support the results of Staley et al. ${ }^{(30)}$ and Bishara et al. ${ }^{(31)}$ who suggested that the malocclusion might tend to minimize or eliminate the differences normally found between the genders.

In general, the findings of Bishara et al. ${ }^{(29)}$ indicated the direction of the post treatment changes were similar in male and female subjects in the various dental arch dimensions evaluated, therefore clinicians should design the retention plan in both male and female subjects, on the basis of characteristics as well as the severity of the original malocclusion rather than on any gender differences. On the other hand, the magnitude of the post treatment changes in some parameters differed significantly between male and female subjects both in extraction and non extraction group, therefore, investigators who are interested in measuring the magnitude of the changes need to treat the data for male and female subjects independently, the result of this study confirm these findings.

\section{CONCLUSIONS}

The extraction and non extraction orthodontic treatment didn't cause narrowing of the maxillary arch in the canine region. Generally, in both genders, most pretreatment arch dimensions were not significantly different between extraction and non extraction groups, while after treatment the extraction group showed reduction in IMW (MBCT), VMD, C-ML, In$\mathrm{ML}$, and A per. Where as the non extraction showed a significant increase in most maxillary arch dimensions. This leads to the conclusion that the kind of treatment may affect the maxillary arch dimensions. The non extraction group had a larger number of significant differences between females and males in the various maxillary arch dimensions than the extraction group, this indicates that the severity of malocclusion might tend to minimize or eliminate the differences normally found between the genders. 


\section{REFERENCES}

1. Bishara SE, Jakobsen JR, Trederd, Nowak A. Arch width changes from 6 weeks to 45 years of age. Am J Orthod Dentofacial Orthop. 1997; 111:401-409.

2. Paquette DE, Beattie JR, Johnston LE Jr. A long term comparison of non extraction and premolar extraction edgewise therapy in "border line" class II patients. Am J Orthod Dentofacial Orthop. 1992; 102: 1 $-14$.

3. Beattie JR, Paqutte DE, Johnston LE Jr. The functional impact of extraction and non extraction treatment: A long - term comparison in patients with "border line", equally susceptible class II malocclusions. Am J Orthod Dentofacial Orthop. 1994; 105:441-449.

4. Saelens NA, DeSmit AA. Theraputic changes in extraction versus non extraction orthodontic treatment. Eur J Orthod. 1998; 20(3):225- 236.

5. Shearn BN, Woods MG. An occlusal and cephalometric analysis of lower first and second premolar extraction effects. Am $J$ Orthod Dentofacial Orthop. 2000; 117(3): 351-361.

6. Ong HB, Woods MG. An occlusal and cephalometric analysis of maxillary first and second premolar extraction effects. Angle Orthod. 2001; 71: 90-102.

7. Gianelly AA. Arch width after extraction and non extraction treatment. Am J Orthod Dentofacial Orthop. 2003; 123:25-28.

8. Kim E, Gianelly AA. Extraction vs non extraction arch widths and smile esthetics. Angle Orthod. 2003; 73:354-358.

9. Dostalova T, Racek D, Tauferova E, Smutny V. Average arch widths and associated changes between initial, post treatment and post- retention measurement. Braz Dent J. 2004; 15(3):8112-8113.

10. Rheude B, Sadowsky L, Ferrera A, Jarobson A. An evaluation of the use of digital study models in orthodontic diagnosis and treatment planning. Angle Orthod. 2004; 75(3):292-296.

11. Hayasaki SM, Castanha Henriques JF, Janson G, de Freitas MR. Influence of extraction and non extraction orthodontic treatment in Japanese- Brazilians with class I and class II division 1 malocclusion. Am J Orthod Dentofacial Orthop. 2005; 127(1):30-36.

12. Ward DE, Workman J, Brown R, Rich- mond S. Change in arch width. Angle Orthod. 2005; 76(1):6-13.

13. Kandasamy S, Woods MG. Is orthodontic treatment without premolar extractions always non extraction treatment. Austral Dent J. 2005; 50(3):146-151.

14. Erdinc AE, Nanda RS, Isiksal E. Relapse of anterior crowding in patients treated with extraction and non extraction of premolars. Am J Orthod Dentofacial Orthop. 2006; 129(6):775-784.

15. Basciftic FA, Usumez S. Effects of extraction and non extraction treatment on class I and class II subjects. Angle Orthod. 2003; 73(1): 36-42.

16. BeGole EA, Fox DL, Sadowsky C. Analysis of change in arch form with premolar expansion. Am J Orthod Dentofacial Orthop. 1998; 113:307-315.

17. Luppanappornlarp S, Johnston LE Jr. The effects of premolar extraction, a long term comparison of outcomes in "clear-cut" extraction and non extraction class II patients. Angle Orthod. 1993; 64:257-272.

18. Bishara SE, Cummin DM, Zaher AR. Treatment and post treatment changes in patients with class II division 1 malocclusion after extraction and non extraction treatment. Am J Orthod Dentofacial Orthop. 1997; 111:18-27.

19. Cross GA, Brooks CN, Lindauer SJ. Arch dimension changes in orthodontic treatment during the 1980s and 1990s in orthodontic treatment effects I. San Diego Convention Center Exhibit Hall C.Poster session. 2002; Pp:6-9.

20. Aksu M, Kocaderli I. Arch width changes in extraction and non extraction treatment in class I patients. Angle Orthod. 2004; 75(6): 948-952.

21. Isik F, Sayinsu k, Nalbantgil D, Arun T. A comparative study of dental arch widths: extraction and non extraction treatment. Eur J Orthod. 2005; 27(6):585589.

22. Kahl - Nieke B, Fischbach H, Schwaze $\mathrm{CW}$. Treatment and post retention changes in dental arch width dimensions a long term evaluation of influencing cofactors. Am J Orthod Dentofacial Orthop. 1996; 109:368-378.

23. De La Cruz AR, Sampson P, Little RM, Artun J, Shapiro PA. Long-term changes in arch form after orthodontic treatment and retention. Am J Orthod Dentofacial 
Orthop. 1995; 107:518-530.

24. Eid AA, Namrawy MM, Kadry WA. The relationship between the width, depth, and circumference of dental arch for a group of Egyptian school children. Egy $J$ orthod. 1987; 85(1):83-87.

25. Younis SAS. Maxillary arch dimensions in Saudi and Egyptian population sample. Am J Orthod. 1984; 1(2):113-137.

26. Mohammad IS. Maxillary arch dimensions: A cross sectional study between 917 years. Master Thesis, Baghdad University- Iraq; 1993.

27. Strang RHW. Factors associated with successful orthodontic treatment. Am J Orthod. 1952; 38:790-800.

28. Boley JC, Mark JA, Sachdeva RC, Buschang PH. Long term stability of class I premolar extraction treatment. Am J Or- thod Dentofacial Orthop. 2003; 124:277287.

29. Bishara SE, Bayati P, Zaher AR, Jakobsen JR. Comparisons of the dental arch changes in patients with class II division 1 malocclusion: extraction vs non extraction treatments. Angle Orthod. 1994; 64(5): 351-358.

30. Staley RN, Stuntz WR, Peterson LC. A comparison of arch widths in adults with normal occlusion and adults with class II malocclusion. Am J Orthod. 1985; 88:163-169.

31. Bishara SE, Jakobsen JR, Angelakis D. Post treatment changes in male and female patients: A comparative study. Am J Orthod Dentofacial Orthop. 1996; 110:624629. 